\title{
Correction to: The Adapted ADOS: A New Module Set for the Assessment of Minimally Verbal Adolescents and Adults
}

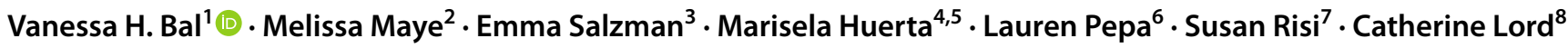

Published online: 30 January 2021

○) Springer Science+Business Media, LLC, part of Springer Nature 2021

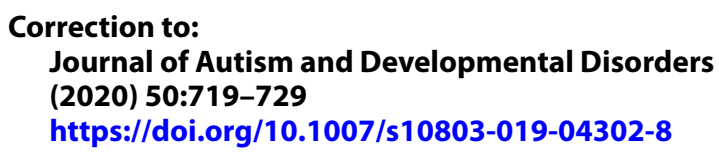

The original version of the article has contained an error in Table 3 (the organization of Module 2 items into the Basic Social Communication and Interaction Quality factors was incorrect).

The updated Table 3 is given below and it shows the correct organization (7 Basic and 3 Interaction Quality items).

Publisher's Note Springer Nature remains neutral with regard to jurisdictional claims in published maps and institutional affiliations.

The original article can be found online at https://doi.org/10.1007/ s10803-019-04302-8.

Vanessa H. Bal

Vanessa.Bal@Rutgers.edu

1 Graduate School of Applied \& Professional Psychology, Rutgers University-New Brunswick, 607 Allison Road, Smithers Hall, Piscataway, NJ 08854-8001, USA

2 Center for Health Policy and Health Services Research, Henry Ford Health System, Detroit, MI, USA

3 UCSF Weill Institute for Neurosciences, University of California, San Francisco, San Francisco, CA, USA

4 Weill Cornell Medicine, New York, NY, USA

5 Felicity House, New York, NY, USA

6 Long Island City, New York, NY, USA

7 Washtenaw Intermediate School District, Early On, Ann Arbor, MI, USA

8 Semel Institute for Neuroscience and Human Behavior, David Geffen School of Medicine, University of California, Los Angeles, Los Angeles, CA, USA 
Table 3 A-ADOS algorithm mapping

\begin{tabular}{|c|c|c|c|c|c|c|c|}
\hline Domains & $\begin{array}{l}\text { Mod 1 FNW } \\
(\mathrm{N}=79)\end{array}$ & $\begin{array}{l}\text { Factor } \\
\text { loadings }\end{array}$ & $\begin{array}{l}\text { Mod 1 SW } \\
(\mathrm{N}=58)\end{array}$ & $\begin{array}{l}\text { Factor } \\
\text { loadings }\end{array}$ & & $\begin{array}{l}\text { Mod 2 } \\
(\mathrm{N}=70)\end{array}$ & $\begin{array}{l}\text { Factor } \\
\text { loadings }\end{array}$ \\
\hline \multirow{10}{*}{$\begin{array}{l}\text { Social } \\
\text { affect }\end{array}$} & Pointing & 0.79 & Initiation of JA & 0.76 & \multirow{7}{*}{$\begin{array}{l}\text { Basic } \\
\text { Soc- } \\
\text { Comm }^{\text {b }}\end{array}$} & Initiation of JA & 0.81 \\
\hline & Gestures & 0.55 & Gestures & 0.40 & & Gestures & 0.23 \\
\hline & $\begin{array}{l}\text { Unusual eye } \\
\text { Contact }\end{array}$ & 0.58 & $\begin{array}{l}\text { Unusual eye } \\
\text { Contact }\end{array}$ & 0.82 & & $\begin{array}{l}\text { Unusual eye } \\
\text { Contact }\end{array}$ & 0.49 \\
\hline & $\begin{array}{l}\text { Facial } \\
\text { Expressions }\end{array}$ & 0.69 & $\begin{array}{l}\text { Facial } \\
\text { expressions }\end{array}$ & 0.53 & & $\begin{array}{l}\text { Facial } \\
\text { expressions }\end{array}$ & 0.49 \\
\hline & $\begin{array}{l}\text { Shared } \\
\text { enjoyment }\end{array}$ & 0.73 & $\begin{array}{l}\text { Shared } \\
\text { enjoyment }\end{array}$ & 0.74 & & $\begin{array}{l}\text { Shared } \\
\text { enjoyment }\end{array}$ & 0.75 \\
\hline & $\begin{array}{l}\text { Amount social } \\
\text { overtures }\end{array}$ & 0.87 & $\begin{array}{l}\text { Amount social } \\
\text { overtures }\end{array}$ & 0.77 & & $\begin{array}{l}\text { Amount social } \\
\text { overtures }\end{array}$ & 0.78 \\
\hline & $\begin{array}{l}\text { Frequency of } \\
\text { vocalization }\end{array}$ & 0.58 & $\begin{array}{l}\text { Amount social } \\
\text { communication }\end{array}$ & 0.83 & & $\begin{array}{l}\text { Amount social } \\
\text { communication }\end{array}$ & 0.61 \\
\hline & Showing & 0.77 & Showing & 0.49 & \multirow{3}{*}{$\begin{array}{l}\text { Inter- } \\
\text { action } \\
\text { quality }^{b}\end{array}$} & $\begin{array}{l}\text { Quality of social } \\
\text { overtures }\end{array}$ & 0.86 \\
\hline & $\begin{array}{l}\text { Social } \\
\text { engagement }\end{array}$ & 0.87 & $\begin{array}{l}\text { Social } \\
\text { engagement }\end{array}$ & 0.92 & & $\begin{array}{l}\text { Quality of social } \\
\text { response }\end{array}$ & 0.57 \\
\hline & & & $\begin{array}{l}\text { Quality of } \\
\text { rapport }\end{array}$ & 0.96 & & $\begin{array}{l}\text { Quality of } \\
\text { rapport }\end{array}$ & 0.92 \\
\hline $\begin{array}{l}\text { Eigen } \\
\text { value }\end{array}$ & 5.42 & & 1.77 & & & $4.94,2.28$ & \\
\hline \multirow{6}{*}{$\begin{array}{l}\text { Restricted } \\
\text { repetitive } \\
\text { behaviors }\end{array}$} & $\begin{array}{l}\text { Repetitive } \\
\text { interests }^{\mathrm{a}}\end{array}$ & 0.12 & $\begin{array}{l}\text { Repetitive } \\
\text { interests }^{\mathrm{a}}\end{array}$ & -0.08 & & $\begin{array}{l}\text { Repetitive } \\
\text { interests }\end{array}$ & 0.49 \\
\hline & $\begin{array}{l}\text { Hand } \\
\text { mannerisms }\end{array}$ & 0.45 & $\begin{array}{l}\text { Hand } \\
\text { mannerisms }\end{array}$ & 0.51 & & $\begin{array}{l}\text { Hand } \\
\text { mannerisms }\end{array}$ & 0.81 \\
\hline & $\begin{array}{l}\text { Odd } \\
\text { Vocalizations }\end{array}$ & 0.93 & $\begin{array}{l}\text { Odd } \\
\text { vocalizations }\end{array}$ & 0.91 & & $\begin{array}{l}\text { Speech } \\
\text { abnormalities }\end{array}$ & 0.40 \\
\hline & $\begin{array}{l}\text { Frequency } \\
\text { undirected Voc. }\end{array}$ & 0.70 & $\begin{array}{l}\text { Frequency } \\
\text { undirected Voc. }\end{array}$ & 0.85 & & $\begin{array}{l}\text { Stereotyped } \\
\text { language }\end{array}$ & 0.64 \\
\hline & \multirow{2}{*}{$\begin{array}{l}\text { Unusual sensory } \\
\text { interest } \\
\text { Unusual facial } \\
\text { expressions }\end{array}$} & 0.44 & $\begin{array}{l}\text { Unusual sensory } \\
\text { interest }\end{array}$ & \multirow[t]{2}{*}{0.30} & & $\begin{array}{l}\text { Unusual sensory } \\
\text { interest }\end{array}$ & \multirow[t]{2}{*}{0.53} \\
\hline & & 0.85 & & & & & \\
\hline $\begin{array}{l}\text { Eigen } \\
\text { value }\end{array}$ & 2.89 & & 7.45 & & & 1.81 & \\
\hline RMSEA & 0.03 & & 0.04 & & & 0.07 & \\
\hline Rho & 0.08 & & 0.50 & & & $0.33,0.31$ & \\
\hline
\end{tabular}

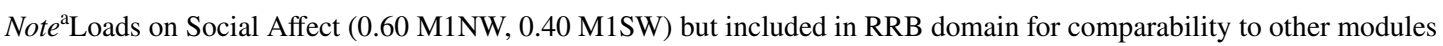

${ }^{\mathrm{b}}$ Module 2 Exploratory Factor Analysis indicated a 3-factor solution dividing Social and Communication items into two factors

$F N W$ few to no words, $S W$ some words, RMSEA root mean square error from exploratory factor analysis (values .08 or less indicate a good fit), Rho correlation between Social Affect \& Restricted Repetitive Behaviors factors for Module 1 and between Basic Social-Communication and Restricted Repetitive Behaviors and Interaction Quality and Restricted Repetitive Behaviors for Module 2, respectively. Rho for Basic and Interaction factors $=0.22$, JA Joint Attention, Frequency Undirected Voc. frequency of undirected vocalizations 